\title{
Catheter malposition during a direct radionuclide cystography — case report
}

Shirin Shahlaei, Farnaz Nesari Javan, Atena Aghaee, Ramin Sadeghi iD

Nuclear Medicine Research Center, School of Medicine, Mashhad University of Medical Sciences, Mashhad, Iran

[Received 1 XII 2019; Accepted 18 VI 2020]

\section{Abstract}

We reported a 15-year-old girl with a history of mild left vesicoureteral reflux who underwent direct radionuclide cystography in our department. Bladder catheterization was mistakenly placed in the vagina. The filling phase showed vagina and uterine cavity which was similar to vesicoureteral reflux. The procedure was repeated with correct catheterization of the bladder and no vesicoureteral reflux was noted.

KEY words: vesicoureteral reflux; direct radionuclide cystography; vagina

Nucl Med Rev 2020; 23, 2: 103-104
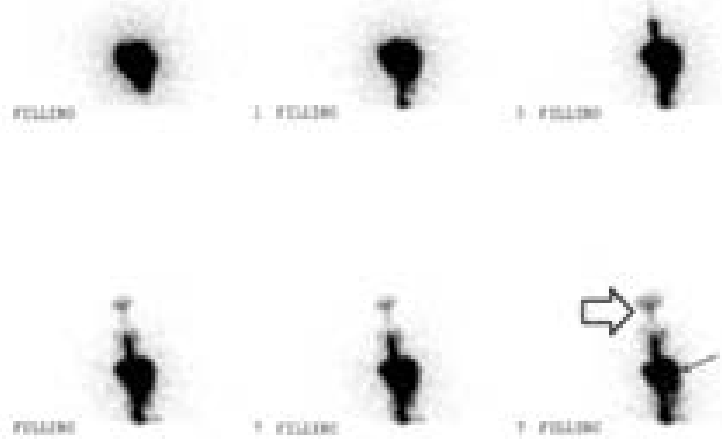

Figure 1. A 15-year-old girl with a previously diagnosed mild left vesicoureteral reflux (VUR) in childhood with history of follow up during previous years without any episode of urinary tract infection, was referred to our nuclear medicine center for direct radionuclide cystography (DRC). She was catheterized under the aseptic conditions using a urinary catheter appropriate for her age then one $\mathrm{mCi}$ of ${ }^{99 \mathrm{mTC}}$ - pertecnetate was injected directly into the catheter and normal saline solution was used to fill the urinary bladder. The patient lied in supine position, and dynamic images were obtained (5 seconds per frame using a dual-head variable angle gamma camera and $128 \times 128$ matrix equipped with low-energy high-resolution collimator) from the posterior view. The images showed a reverse pear shape of tracer accumulation with two abnormal linear tracer uptakes in the midline similar to vesicoureteral reflux. We checked the catheter and it was mistakenly placed in the vagina and unusual distribution of the tracer was due to tracer flow to the vagina (arrow) and uterine cavity (arrow head)

Correspondence to: Ramin Sadeghi

Nuclear Medicine Research Center, School of Medicine, Mashhad University of Medical Sciences, Mashhad, Iran

e-mail: sadeghir@mums.ac.ir
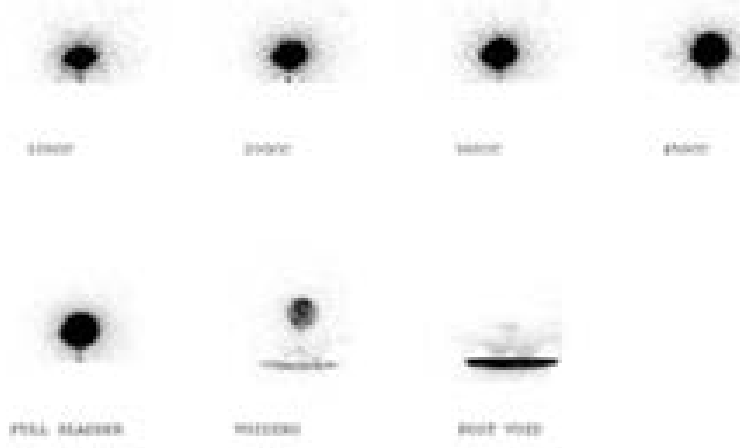

Figure 2. The catheter was removed and the correct catheterization was done. Filling phase and voiding imaging were done with a similar protocol. No VUR was shown in images

A major health problem in childhood is vesicoureteral reflux (VUR) [1]. Since VUR may have negative effects on kidneys it is important to detect the VUR as soon as possible [2]. In this regard voiding cystourethrography (VCUG) and direct radionuclide cystography (DRC) are common methods to diagnose and follow VUR $[1,3]$. Voiding cystourethrography (VCUG) have some disadvantages i.e. high gonadal radiation compared with DRC [4]. Instead, direct radionuclide cystography (DRC) has been proposed to detect VUR with better detection of intermittent reflux. However, this method is suffering from the lack of enough anatomical details [5-7]. Our case report showed the importance of careful attention to correct catheterization (Fig. 1 and 2). Catheter 
insertion in the vagina has been reported before during VCUG and this pitfall should always be borne in mind in case of DRC procedure especially in those with an unusual pattern of tracer distribution.

\section{References}

1. Joaquim Al, de Godoy MF, Burdmann EA. Cyclic Direct Radionuclide Cystography in the Diagnosis and Characterization of Vesicoureteral Reflux in Children and Adults. Clin Nucl Med. 2015; 40(8): 627-631, doi: 10.1097/RLU.0000000000000799, indexed in Pubmed: 26018721.

2. Lebowitz RL, Lebowitz RL. The detection of vesicoureteral reflux in the child. Invest Radiol. 1986; 21(7): 519-531, doi: 10.1097/00004424-19860700000003, indexed in Pubmed: 3015828.

3. Massoudi T, Shayegani H, Sadeghi R. Radiotracer Injection Into the Catheter Balloon: A Subtle Pitfall Which Can Be Overlooked in Direct
Radionuclide Cystography. Clin Nucl Med. 2017; 42(4): 289-292, doi: 10.1097/RLU.0000000000001539, indexed in Pubmed: 28121664.

4. Zerin JM, Lebowitz RL. Catheter malposition during cystography: a cause of diagnostic errors. AJR Am J Roentgenol. 1989; 153(2): 363-367, doi: 10.2214/ajr.153.2.363, indexed in Pubmed: 2750622.

5. Fettich J, Colarinha P, Fischer S, et al. Empfehlungen zur Durchführung der direkten Radionuklid-Zystographie bei Kindern - Richtlinie übernommen vom Paediatric Committee der European Association of Nuclear Medicine (EANM). Der Nuklearmediziner. 2002; 25(2): 106-111, doi: 10.1055/s2002-33278.

6. Mandell GA, Eggli DF, Gilday DL, et al. Procedure guideline for radionuclide cystography in children. Society of Nuclear Medicine. J Nucl Med. 1997; 38(10): 1650-1654, indexed in Pubmed: 9379209.

7. Polito C, Moggio G, La Manna A, et al. Cyclic voiding cystourethrography in the diagnosis of occult vesicoureteric reflux. Pediatr Nephrol. 2000; 14(1): 39-41, doi: 10.1007/s004670050010, indexed in Pubmed: 10654329. 